\title{
ON THE POINT SPECTRUM OF A TOEPLITZ OPERATOR
}

\author{
BY \\ DOUGLAS N. CLARK $\left({ }^{1}\right)$
}

0. Introduction. Let $1 \leqq p \leqq \infty, L^{p}=L^{p}(0,2 \pi)$ and $H^{p}$ the set of periodic functions $x^{+}(\phi) \in L^{p}$ with Fourier coefficients vanishing on the negative integers: $x^{+}(\phi) \sim \sum_{n=0}^{\infty} x_{n} e^{i n \phi}$.

Let $f(\phi) \in L^{q}, 1 / p+1 / q=1$. Then, for any $x^{+}(\phi) \in H^{p}, f(\phi) x^{+}(\phi) \in L^{1}$ and therefore formally $f(\phi) x^{+}(\phi) \sim y^{+}(\phi)+\bar{v}^{+}(\phi) e^{-i \phi}$. We define the Toeplitz operator $T=T(f): H^{p} \rightarrow H^{p}$ associated with $f$, by

$$
\begin{gathered}
D(T(f))=\left\{x^{+}(\phi) \in H^{p}:\right. \\
\left.f(\phi) x^{+}(\phi)=y^{+}(\phi)+\bar{v}^{+}(\phi) e^{-i \phi} \text { and } y^{+} \in H^{p}\right\} \\
y^{+}(\phi)=T(f) x^{+}(\phi) .
\end{gathered}
$$

Below, we assume that $q \geqq 2$ (so that $1<p \leqq 2$ and $H^{p} \supset H^{q}$ ) to assure that $D(T(f)$ ) contains all polynomials in $e^{i \phi}$ and is dense in $H^{p}$. (We insist that $f \in L^{q}, 1 / p+1 / q=1$, so that $f x \in L^{1}$, for $x \in D(T(f))$.) It should be noted that the results below remain valid if $p$ is replaced by any index $p^{\prime}, p \leqq p^{\prime} \leqq 2$, since $f(\phi) \in L^{q^{\prime}}$ for $2 \leqq q^{\prime} \leqq q$.

In [4], for $p=2$, Hartman and Wintner posed the problem of the determination of the location of the spectrum and point spectrum of $T(f)$. A number of steps ([6], [9], [1], [12], [2], [3], [8]) toward solving this problem have been taken. In some investigations of the point spectrum, additional assumptions have been made, such as, for example, that $T(f)$ is selfadjoint [6], that $\sum\left|f_{n}\right|<\infty$ ([9], [3]), or that $f(\phi)$ is bounded and satisfies

$$
\arg f(\phi)=g(\phi)+h(\phi)
$$

where $g(\phi)=\sum_{j=1}^{n} \alpha_{j} J\left(\phi-\theta_{j}\right), J(\phi)=\phi-2 \pi[\phi / 2 \pi],[x]$ the integral part of $x$, and the conjugate of $h(\phi)=$ conj $h(\phi) \in L^{\infty}$ [12]. In [7], Hartman found necessary and sufficient conditions that 0 (and hence any complex $\lambda$ ) belong to the point spectrum of $T(f), p=2$. Hartman's general conditions are in terms of the conj $\arg f(\phi)$.

If $N(T(f))$ is the null space of $T(f)$, we find necessary and sufficient conditions on $f(\phi)$ that $\operatorname{dim} N(T(f))=m$ (Theorem 1.1) under the assumption that $(0.1)$ holds with $h(\phi)$ continuous. This result is in terms of conj $h(\phi)$. When it is assumed, for example, that conj $h(\phi)$ is bounded (Corollary 1.2), our results generalize those of [12]. We make no use of the hypothesis $\|f\|_{\infty}<\infty$ and, even in case $f(\phi)$ is bounded, our results are more general than those of [12].

Received by the editors March 30, 1966.

( ${ }^{1}$ This work was supported by the Air Force Office of Scientific Research. 
Our methods are based on those developed in connection with the counterexample presented in [6]. Namely, for $f$ real valued, for example, we first build solutions $x^{+}(\phi), y^{+}(\phi) \in H^{2}$ of

$$
\begin{gathered}
(\operatorname{sgn} f(\phi)) \bar{x}^{+}(\phi) e^{-i \phi} \in H^{1}, \\
|f(\phi)| \bar{y}^{+}(\phi) \in H^{1}
\end{gathered}
$$

and then decide when $x^{+} y^{+} \in H^{2}$. Actually, the existence part of our Theorem 1.1 follows directly from Hartman's theorem [7]. In order to prove that we have found all eigenvectors, however, we use the equations (0.2). Having found conditions for $\operatorname{dim} N(T(f))=m$ in terms of conj $h(\phi)$ (Theorem 1.1), we proceed along the lines of [12] and [7] to remove the term conj $h(\phi)$ from our conditions in some cases.

The novel ideas introduced in this paper, which permit us to obtain some generalizations of results of [7], [12], are the notion of the multiplicity $M_{\gamma}^{p}(f, \theta, H)$ introduced in $\$ 1$ and the operator $T_{0}^{0}(f)$ in Lemma 1.2.

When applied to real-valued $f(\phi)$ (Corollary 1.1), our theorems locate the real part of the point spectrum of $T(f)$ (assuming $\operatorname{sgn}(f(\phi)-\lambda)$ has a finite number of discontinuities for all real $\lambda$ ). When applied to the question of selfadjointness of $T(f)$ for real-valued $f$ (Theorem 2.1), our results generalize those of [7] and answer a question raised there.

Of the papers mentioned above which deal with the point spectrum of $T=T(f)$, [9] and [3] deal with $T$ as a "matrix" acting on the $l^{p}$ sequence spaces $1 \leqq p \leqq \infty$ (rather than on the $H^{p}$-spaces). Under the conditions imposed upon $f(\phi)$ there, the null-space of $T(f)$ was found to be independent of $p$. Our results show that if $T(f)$ is considered as an operator on $H^{p^{\prime}}$, where $p \leqq p^{\prime} \leqq 2$, then the dimension of the null-space of $T(f)$ can depend on $p^{\prime}$. Actually, if we impose the conditions of [3] (i.e., $e^{i N \phi} f(\phi)$ is a polynomial in $e^{i \phi}$ for some positive integer $N$ ) or [9] (i.e., $0<c \leqq f(\phi) \leqq C)$ upon $f(\phi)$, in addition to our own, we see that $\operatorname{dim} N(T)$, for $T$ acting on $H^{p^{\prime}}$-spaces is the same for $p \leqq p^{\prime} \leqq 2$.

1. Basic theorems. First, we make the following

Definition. Let $f(\phi) \in L^{q}, 0 \leqq \theta \leqq 2 \pi,-1<\gamma \leqq 0$, and let $H(\phi)$ be a measurable periodic function of period $2 \pi$. We say that

$$
e^{H(\phi)}|\phi-\theta|^{2 \gamma} \mid f(\phi) \notin L^{p / 2}(U)
$$

holds with multiplicity $M=M_{\gamma}^{p}(f, \theta, H)$ if $M=\min \{N: N \geqq 0$, an integer, $e^{H(\phi)}(\phi-\theta)^{2 N+2 \gamma} \mid f(\phi) \in L^{p / 2}(U)$, for all sufficiently small neighborhoods $U$ of $\left.\theta\right\}$, and with multiplicity $M=\infty$ if the above set of integers $N$ is empty. In particular, if (1.1) does not hold for some neighborhood $U$ of $\theta$, then the multiplicity $M=0$.

Following Widom [12], we set

$$
J(\phi)=\phi-2 \pi[\phi / 2 \pi]
$$

for $[x]$ the integral part of $x$. 
THEOREM 1.1. Let $f(\phi) \in L^{q}, q^{-1}+p^{-1}=1, \infty>q \geqq 2$, and let there exist constants $\alpha_{j}$ and $\theta_{j}$ with the property that $0 \leqq \theta_{j}<2 \pi$ and for

$$
g(\phi)=-\sum_{j=1}^{n} \alpha_{j} J\left(\phi-\theta_{j}\right)
$$

there is a function

$$
\psi(\phi)=\arg f(\phi)
$$

defined a.e. such that

$$
h(\phi)=\psi(\phi)-g(\phi)
$$

can be extended as a continuous, periodic function, of period $2 \pi$. Let $\alpha_{j}$ have the decomposition

$$
\alpha_{j}=\beta_{j}+\gamma\left(\theta_{j}\right), \quad \beta_{j} \in Z, \quad-1<\gamma\left(\theta_{j}\right) \leqq 0 .
$$

For $0 \leqq \theta<2 \pi$ and $\theta \neq \theta_{j}$, let $\gamma(\theta)=0$. Let

$$
d=\sum_{j=1}^{n} \beta_{j} \quad \text { and } \quad k=\sum_{0 \leqq \theta<2 \pi} M_{\gamma(\theta)}^{p}(f, \theta, \operatorname{conj} h(\phi)) \leqq \infty .
$$

Then, if $\log |f(\phi)| \in L^{1}$ and $k<\infty$,

(i) $d-k>0$ implies that $R(T)$ is dense in $H^{p}$ and $\operatorname{dim} N(T(f))=d-k$.

(ii) $d-k \leqq 0$ implies that $T(f)$ is one-to-one and $R(T)$ contains a polynomial in $e^{i \phi}$ of degree $k-d$, but none of degree $<k-d$. If $k=\infty$, then $T(f)$ is one-to-one and $R(T)$ contains no trigonometric polynomials. Furthermore, $\log |f(\phi)| \notin L^{1}$ and $f \not 0$ imply that $R(T(f)) \neq \bar{R}(T(f))=H^{p}$.

It is understood that the assumption $k=\infty$ includes, in particular, the case in which there are an infinite number of $\theta$ in $0 \leqq \theta<2 \pi$ which satisfy (1.1).

Corresponding questions concerning the codim $R(T)$ will be discussed below.

Proof. Suppose, first, that $\log |f(\phi)| \notin L^{1}$. Now, if $f \not \equiv 0$ and $T(f)$ has a nullspace, an equation of the form $f(\phi) x^{+}(\phi)=e^{-i n \phi}\left(1+\bar{y}^{+}(\phi) e^{-i \phi}\right)$ with $x^{+} \in H^{p}$, $y^{+} \in H^{1}, n>0$, must hold. But then

$$
\log |f(\phi)|=\log \left|\left(1+\bar{y}^{+}(\phi) e^{-i \phi}\right)\right|-\log \left|x^{+}(\phi)\right| \in L^{1} .
$$

Thus $T(f)$ has no null-space. Applying the same argument with $n=0$, shows that $1 \notin R(T(f))$ and hence $R(T(f)) \neq H^{p}$.

If $\bar{R}(T(f)) \neq H^{p}$, there is a $y^{+} \in H^{q}$ satisfying $\left(y^{+}, T(f) p^{+}\right)=0$ for all polynomials $p^{+}(\phi)$ in $e^{i \phi}$. Now $f(\phi) p^{+}(\phi)=T(f) p^{+}+\bar{x}^{+} e^{-i \phi} \in L^{p}$, so that $p>1$ implies that $\bar{x}^{+}(\phi) e^{-i \phi} \in L^{p}$. Since $\left(y^{+}, \bar{x}^{+} e^{-i \phi}\right)=0$, we have $\left(y^{+}, f p^{+}\right)=0$. This means that the Fourier coefficients of the function $z(\phi)=y^{+}(\phi) f(\phi) \in L^{1}$ vanish on the nonnegative integers and $z(\phi) \not \equiv 0$ since $f(\phi) \not \equiv 0$ and $y^{+}(\phi) \neq 0$ almost everywhere. Thus $\log |f(\phi)|=\log |z(\phi)|-\log \left|y^{+}(\phi)\right| \in L^{1}$. 
Hence we have to consider only the case that $\log |f(\phi)| \in L^{1}$. Assume that $k<\infty$. Observe, now, that for $J(\phi)$, we have

$$
\operatorname{conj} \alpha J(\phi-\theta)=-\alpha \log \left|1-e^{i(\phi-\theta)}\right|^{2} .
$$

Thus, for $g(\phi)$, we have

$$
\operatorname{conj} g(\phi)=\log \prod_{j=1}^{n}\left|1-e^{i\left(\phi-\theta_{j}\right)}\right|^{2 \alpha_{j}}
$$

Notice that, since $\alpha_{j}-\beta_{j}=\gamma\left(\theta_{j}\right)$, and since, for a suitable definition of $\arg (\bmod 2 \pi)$, conj $\arg \exp \left[i \sum_{j=1}^{n} \beta_{j}\left(\phi-\theta_{j}\right)\right]=\operatorname{conj} \arg C e^{i d \phi}=-2 \log \prod_{j=1}^{n}\left|1-\exp \left(i\left(\phi-\theta_{j}\right)\right)\right|^{\beta_{j}}$, where the first equality serves to define $C$,

$$
\frac{1}{2} \operatorname{conj}\left[g(\phi)+\arg C e^{i d \phi}\right]=\log \prod_{j=1}^{n}\left|1-e^{i\left(\phi-\theta_{j}\right)}\right|^{\gamma\left(\theta_{j}\right)} .
$$

Thus, for any constant $\alpha$,

$$
G(\phi)=\log \prod_{j=1}^{n}\left|1-\exp \left(i\left(\theta_{j}-\phi\right)\right)\right|^{\gamma\left(\theta_{j}\right)}-\frac{1}{2} i[g(\phi)+d \phi]+\alpha \in H^{1} ;
$$

hence,

$$
F(\phi)=e^{G(\phi)}=\prod_{j=1}^{n}\left|1-\exp \left(i\left(\theta_{j}-\phi\right)\right)\right|^{\gamma(\theta,)} \exp \left[-\frac{1}{2} i(g(\phi)+d \phi)\right]
$$

is an outer function in the sense of Beurling and

$$
\begin{gathered}
\bar{F}(\phi) / F(\phi)=e^{i g(\phi)} e^{i d \phi}, \\
F(\phi) \in \cdot H^{1}, \quad 1 / F(\phi) \in H^{\infty} .
\end{gathered}
$$

Suppose that $\theta_{1}^{\prime}, \ldots, \theta_{k}^{\prime}$ are the points such that (1.1) holds with $\theta=\theta_{j}^{\prime}$, each included as many times as its multiplicity $M_{\gamma(\theta)}^{p}(f, \theta$, conj $h)$. We have

$$
\operatorname{conj} \arg \exp \left[i \sum_{j=1}^{k}\left(\phi-\theta_{j}^{\prime}\right)\right]=\operatorname{conj} \arg C e^{i k \phi}=-2 \log \prod_{j=1}^{k}\left|1-e^{i\left(\phi-\theta_{j}^{j}\right)}\right| ;
$$

so that

$$
F_{1}(\phi)=\prod_{j=1}^{k}\left|1-\exp \left(i\left(\phi-\theta_{j}^{\prime}\right)\right)\right| e^{i k \phi / 2}
$$

is an outer function in $H^{\infty}$ and

$$
\bar{F}_{1}(\phi) / F_{1}(\phi)=e^{-\imath k \phi} .
$$


Since $h(\phi)=\psi(\phi)-g(\phi)$ is a continuous, periodic function,

$$
x_{1}^{+}(\phi)=\exp (1 / 2)(\operatorname{conj} h(\phi)-i h(\phi)) \in H^{b} \quad \text { for } 1 \leqq b<\infty,
$$

and thus

$$
e^{i n(\phi)} x_{1}^{+}(\phi)=\bar{x}_{1}^{+}(\phi),
$$

$$
x_{1}^{+}(\phi), \quad 1 / x_{1}^{+}(\phi) \in H^{b} \quad \text { for } 1 \leqq b<\infty .
$$

Further, we have that $\log |f(\phi)| \in L^{1}$ implies that there is an outer function $1 / x^{+}(\phi) \in H^{2}$ such that

$$
|f(\phi)|=\left(1 / x^{+}(\phi)\right)\left(1 / \bar{x}^{+}(\phi)\right)
$$

([11, p. 235]; take $1 / x^{+}$to be the outer factor of $|f|^{1 / 2}$ as in [7]).

Combining (1.2), (1.3), (1.4) and (1.5), we have, from

$$
f(\phi)=|f(\phi)| \exp i(h(\phi)+g(\phi))
$$

that

$$
f(\phi) e^{i(d-k) \phi}\left[F(\phi) F_{1}(\phi) x^{+}(\phi) x_{1}^{+}(\phi)\right]=\bar{F}(\phi) \bar{F}_{1}(\phi) \bar{x}_{1}^{+}(\phi) / \bar{x}^{+}(\phi) .
$$

Now suppose that, in the set $\left\{\theta_{1}^{\prime}, \ldots, \theta_{k}^{\prime}\right\}$, there are $m$ distinct points, $\theta_{1}^{\prime}, \ldots, \theta_{m}^{\prime}$; further, if $s, 0 \leqq s \leqq m$, of the distinct numbers $\theta_{1}, \ldots, \theta_{n}$ occur in the set $\theta_{1}^{\prime}, \ldots, \theta_{k}^{\prime}$, change the enumerations so that $\theta_{1}^{\prime}=\theta_{1}, \ldots, \theta_{s}^{\prime}=\theta_{s}$. For $j=1, \ldots, m$, let

$$
M_{j}=M_{\gamma\left(\theta_{j}\right)}^{p}\left(f, \theta_{j}^{\prime}, \operatorname{conj} h\right) .
$$

Then we have

$$
\left|F(\phi) F_{1}(\phi) x^{+}(\phi) x_{1}^{+}(\phi)\right|=\frac{e^{(1 / 2) \operatorname{conj} h(\phi)} \prod_{j=1}^{m}\left|1-\exp \left(i\left(\phi-\theta_{j}^{\prime}\right)\right)\right|^{M_{j}+\gamma\left(\theta_{j}^{\prime}\right)}}{\prod_{j=s+1}^{n}\left|1-\exp \left(i\left(\phi-\theta_{j}\right)\right)\right|^{-\gamma\left(\theta_{j}\right)}|f(\theta)|^{1 / 2}} .
$$

Thus $F(\phi) F_{1}(\phi) x^{+}(\phi) x_{1}^{+}(\phi) \in H^{p}$ follows from the fact that, on the one hand, $\theta_{s+1}, \ldots, \theta_{n}$ do not satisfy (1.1), so that, in a sufficiently small neighborhood $U$ of $\theta_{s+1}, \ldots, \theta_{n}, \prod_{j=1}^{m}\left|1-\exp \left(i\left(\phi-\theta_{j}^{\prime}\right)\right)\right|^{M_{j}+\gamma\left(\theta_{j}^{\prime}\right)} \in L^{\infty}$ implies that $F F_{1} x^{+} x_{1}^{+} \in L^{p}(U)$; and that, on the other hand, in a neighborhood $U$ of $\theta_{1}^{\prime}, \ldots, \theta_{m}^{\prime}$, the definition of $M_{j}=M_{\gamma\left(\theta_{j}^{\prime}\right)}^{p}\left(f, \theta_{j}^{\prime}\right.$, conj $\left.h\right)$ implies $F F_{1} x^{+} x_{1}^{+} \in L^{p}(U)$. Now $F F_{1} x^{+} x_{1}^{+} \in H^{p}$ since $F F_{1} x^{+} x_{1}^{+}$is the quotient of the $H^{1}$ function $F_{1} F$ by the $H^{1}$ outer function $1 /\left(x_{1}^{+} x^{+}\right)$.

Thus (1.6) shows that, if $d-k>0, \operatorname{dim} N(T) \geqq d-k$ and if $d-k \leqq 0$, then $R(T)$ contains a polynomial in $e^{i \phi}$ of degree $k-d$.

In order to complete the proof of Theorem 1.1, let $y^{+}(\phi) \in H^{p}$ and $y_{1}^{+}(\phi) \in H^{1}$ satisfy

$$
f(\phi) y^{+}(\phi)=e^{-i N \phi} \bar{y}_{1}^{+}(\phi)
$$

where $N$ is an integer. From (1.4) and (1.5),

$$
|f(\phi)|^{-1} e^{-\operatorname{th}(\phi)}\left(1 / x_{1}^{+}(\phi) x^{+}(\phi)\right)=\bar{x}^{+}(\phi) / \bar{x}_{1}^{+}(\phi),
$$


where $\left|1 / x^{+}(\phi)\right|=|f(\phi)|^{1 / 2} \in L^{2 q}$ and $\left|1 / x_{1}^{+}(\phi)\right| \in L^{b}, 1 \leqq b<\infty$, so that, in particular, $1 /\left|x_{1}^{+}(\phi)\right| \in L^{2 q}$, and thus

$$
\left|1 / x^{+}(\phi) x_{1}^{+}(\phi)\right| \in L^{q} .
$$

Combining this with (1.7) and $f /|f|=e^{i \downarrow}=e^{i(g+h)}$,

$$
e^{i g(\phi)}\left[y^{+}(\phi) / x_{1}^{+}(\phi) x^{+}(\phi)\right]=e^{-i N \phi}\left[\bar{y}_{1}^{+}(\phi) \bar{x}^{+}(\phi) / \bar{x}_{1}^{+}(\phi)\right] .
$$

Thus every solution $y^{+}(\phi) \in H^{p}$ of (1.7) can be written in the form

$$
y^{+}(\phi)=X^{+}(\phi) x_{1}^{+}(\phi) x^{+}(\phi),
$$

where $X^{+}(\phi) \in H^{1}$ is a solution of

$$
\begin{gathered}
e^{i g(\phi)} X^{+}(\phi)=e^{-i N \phi} \bar{Y}^{+}(\phi), \\
e^{(1 / 2) \operatorname{conj} h(\phi)} X^{+}(\phi) /|f(\phi)|^{1 / 2} \in L^{p},
\end{gathered}
$$

since $\left|x^{+}\right|=1 /|f|^{1 / 2}$ and $\left|x_{1}^{+}\right|=\exp \left(\frac{1}{2} \operatorname{conj} h\right)$.

We will need the following analogue of a theorem of Hartman ([7, Corollary 4.3]):

LEMMA 1.1. Let $f_{0}(\phi)$ be a function with a representation

$$
f_{0}(\phi)=e^{-i n \phi} \bar{F}^{+}(\phi) / F^{+}(\phi)
$$

where $F^{+}(\phi) \in H^{1}, 1 / F^{+}(\phi) \in H^{\infty}$, and $n$ is an integer. Then $X^{+}(\phi), 0 \not \equiv X^{+}(\phi) \in H^{1}$, satisfies a relation of the form

$$
f_{0}(\phi) X^{+}(\phi)=e^{-i N \phi} \bar{Y}^{+}(\phi), \quad Y^{+}=\sum_{j=0}^{\infty} Y_{j} e^{i j \phi}, \quad Y_{0} \neq 0 .
$$

if and only if $N \leqq n$; in which case,

$$
X^{+}(\phi)=p^{+}(\phi) F^{+}(\phi),
$$

where $p^{+}(\phi)$ is an arbitrary polynomial in $e^{i \phi}$ of degree exactly $n-N$.

Assume Lemma 1.1 for the moment. Let $y^{+}(\phi)=X^{+}(\phi) x_{1}^{+}(\phi) x^{+}(\phi)$ be a solution of (1.7), where $X^{+}(\phi)$ satisfies (1.8). Let $f_{0}(\phi)=e^{i g(\phi)}, F^{+}(\phi)=F(\phi), n=d$ in Lemma 1.1 ; cf. (1.2). Now if $d>k$, we let $N \geqq 1$, and Lemma 1.1 implies that

$$
X^{+}(\phi)=p^{+}(\phi) F^{+}(\phi)
$$

where $p^{+}$is a polynomial in $e^{i \phi}$ of degree at most $d-1$. But $\left.\left.\left|e^{(1 / 2) \operatorname{conj} h(\phi)} X^{+}(\phi) /\right| f(\phi)\right|^{1 / 2}|=| p^{+}(\phi) e^{(1 / 2) \operatorname{conj} h(\phi)} F(\phi)|/| f(\phi)\right|^{1 / 2}$

$$
=\left.\left|p^{+}(\phi)\right|\left|e^{(1 / 2) \operatorname{conj} h(\phi)}\right| \prod_{j=1}^{n}\left|1-\exp \left(i\left(\theta_{j}-\phi\right)\right)\right|^{\gamma\left(\theta_{j}\right)}|| f(\phi)\right|^{1 / 2} .
$$


The condition $x_{1}^{+}(\phi) X^{+}(\phi) /|f(\phi)|^{1 / 2} \in L^{p}$ in (1.8) implies that $p^{+}(\phi)=q^{+}(\phi) p_{1}(\phi)$, where $q^{+}(\phi)$ is a polynomial in $e^{i \phi}$ of degree (at most) $d-k-1$,

$$
p_{1}(\phi)=\prod_{j=1}^{m}\left|1-\exp \left(i\left(\phi-\theta_{j}^{\prime}\right)\right)\right|^{M_{j}}, \quad \operatorname{deg} p_{1}=k,
$$

and $\theta_{1}^{\prime}, \ldots, \theta_{m}^{\prime}, M_{j}$ are defined after (1.6).

Thus, every solution $y^{+}$of (1.7) can be written in the form

$$
y^{+}(\phi)=\left(x_{1}^{+}(\phi) x^{+}(\phi) p_{1}(\phi) F(\phi)\right) q^{+}(\phi)
$$

where $q^{+}$is some polynomial in $e^{i \phi}$ of degree $d-k-1$. Hence, $\operatorname{dim} N(T)^{\prime} \leqq d-k$. This proves Theorem 1.1 in case $d-k>0$.

In case $k \geqq d$, suppose that $R(T)$ contains a polynomial in $e^{i \phi}$ of degree less than $k-d$. Lemma 1.1 , with $N \leqq d-k+1$, implies that

$$
X^{+}(\phi)=p^{+}(\phi) F^{+}(\phi)
$$

where the degree of $p^{+}$is at most $k-1$. But from the above considerations, it is clear that the second line of (1.8) cannot hold if $p^{+}$is of degree less than $k$.

Consider the case $k=\infty$. Since (1.2), (1.4), and (1.5) did not involve the assumption $k \leqq \infty,(1.7)$ still implies (1.8). If (1.7), and hence the first line of (1.8) holds, then, from Lemma 1.1 with $f_{0}(\phi)=e^{i g(\phi)}, F^{+}(\phi)=F(\phi)$ and $N<1$, we conclude that $X^{+}(\phi)=p^{+}(\phi) F(\phi)$ where $p^{+}$is a polynomial in $e^{i \phi}$. But, for $k=\infty$, and $p^{+}$a polynomial, the second line of (1.8) cannot hold. Thus (1.7) cannot hold. This completes the proof of Theorem 1.1, assuming Lemma 1.1.

Proof of Lemma 1.1. In case $N \leqq n$, it is clear that $X^{+}(\phi)=e^{i(n-N) \phi} F^{+}(\phi)$ satisfies the desired type of relation.

Conversely, if $X^{+}(\phi) \in H^{1}$ satisfies

$$
f_{0}(\phi) X^{+}(\phi)=e^{-i N \phi} \bar{Y}^{+}(\phi)
$$

for $N>n$, then $\left(e^{i n \phi} f_{0}(\phi) X^{+}(\phi), b^{+}(\phi)\right)=0$ for all $b^{+}(\phi) \in H^{\infty}$; in particular, for $b^{+}(\phi)=e^{i j \phi} / F^{+}(\phi), j \geqq 0$. Thus

$$
\begin{aligned}
\left(e^{i n \phi} f_{0}(\phi) X^{+}(\phi), e^{i j \phi} / F^{+}(\phi)\right) & =\left(X^{+}(\phi) \bar{F}^{+}(\phi) / F^{+}(\phi), e^{i j \phi} / F^{+}(\phi)\right) \\
& =\left(X^{+}(\phi) / F^{+}(\phi), e^{i j \phi}\right)=0
\end{aligned}
$$

But this, together with $X^{+}(\phi) / F^{+}(\phi) \in H^{1}$ implies $X^{+}(\phi) \equiv 0$. Thus $N \leqq n$.

In order to complete the proof of the lemma, we assume $N=0, n \geqq 0$ (by replacing $n$ by $n-N$, if necessary) and use induction on $n$. If $n=0$, suppose that $Z^{+}(\phi) \in H^{1}$ satisfies

$$
Z^{+}(\phi) f_{0}(\phi)=\bar{Y}^{+}(\phi), \quad Y_{0} \neq 0
$$


Write $F^{+}=\sum_{n=0}^{\infty} p_{n} e^{i n \phi}$, so that $F^{+}$is outer implies that $p_{0} \neq 0$. Then

$$
Z^{+}(\phi)-Y_{0} F^{+}(\phi)
$$

satisfies

$$
f_{0}(\phi)\left(p_{0} Z^{+}(\phi)-Y_{0} F^{+}(\phi)\right)=p_{0} \bar{Y}^{+}(\phi)-Y_{0} \bar{F}^{+}(\phi)=e^{-i \phi} \bar{W}^{+}(\phi) .
$$

Thus $p_{0} Z^{+}(\phi)-Y_{0} F^{+}(\phi) \equiv 0$ by the first part of the lemma. In the case in which $n=n_{0}>0$, notice that

$$
X^{+}(\phi)=F^{+}(\phi)\left(e^{i n \phi}+p^{+}(\phi)\right)
$$

is a solution where $p^{+}(\phi)$ is any polynomial in $e^{i \phi}$ of degree less than $n_{0}$. Thus the statement that there is a solution $Z^{+}(\phi)$ which cannot be written in the desired form is equivalent to the statement that there are $n_{0}+2$ linearly independent solutions. This case is easily reduced to the case in which $n \leqq n_{0}-1$ and there are $n_{0}+1$ solutions. This proves Lemma 1.1.

Definition. For $2 \leqq q<\infty$, and $f(\phi) \in L^{q}$, the operator $T^{0}(f): H^{q} \rightarrow H^{q}$ will mean the operator with domain $D^{0}=\left\{x^{+}(\phi) \in H^{q}: x^{+}(\phi) f(\phi) \in H^{1}\right.$ satisfies $\left.x^{+}(\phi) f(\phi)=y^{+}(\phi)+e^{-i \phi} \bar{y}_{1}^{+}(\phi), y^{+}(\phi) \in H^{q}\right\}$ defined by $T^{0}(f) x^{+}=y^{+}$. Let $T_{0}^{0}(f)$ denote the restriction of $T^{0}(f)$ to $D_{0}^{0}=\left\{x^{+}(\phi) \in H^{q}: x^{+}(\phi) f(\phi) \in L^{q}\right\}$, and $T_{0}(f)$, the restriction of $T^{0}(f)$ to $D_{0}$, the linear manifold of polynomials in $e^{i \phi}$. Note that $T_{0}^{0}$ is well defined, since, for $1<q<\infty$, the conjugate of an $L^{q}$ function also belongs to $L^{q}$.

Thus, for $f(\phi) \in H^{q}, 2 \leqq q<\infty$, we have associated the five operators: the Toeplitz operator $T(f): H^{p} \rightarrow H^{p}, 1 / p+1 / q=1$, its adjoint $T(f)^{*}: H^{q} \rightarrow H^{q}$ and the operators $T^{0}(f): H^{q} \rightarrow H^{q}, T_{0}^{0}(f): H^{q} \rightarrow H^{q}$ and $T_{0}(f): H^{q} \rightarrow H^{q}$.

In case $p=q=2, T^{0}(f)=T(f)$ and the operator $T(f)^{*}$ is the closure of $T_{0}(f)$ [6, p. 878]. One sees precisely in the same manner that, in the case of

$$
T(\bar{f}): H^{p} \rightarrow H^{p}, \quad 1<p \leqq 2,
$$

the adjoint operator $T(\bar{f})^{*}: H^{q} \rightarrow H^{q}, 1 / p+1 / q=1$, is the closure of

$$
T_{0}(f): H^{q} \rightarrow H^{q} .
$$

LEMMA 1.2. Let $f(\phi) \in L^{q}, 2 \leqq q<\infty$, then

$$
T_{0}(f) \subset T_{0}^{0}(f) \subset T(f)^{*} \subset T^{0}(f) .
$$

In particular, $D^{0} \supset D\left(T(\bar{f})^{*}\right) \supset D_{0}^{\circ}$.

Note, since $T(f)^{*}$ is the closure of $T_{0}^{0}(f)$, that, whenever $T_{0}^{0}(f)$ is a closed operator (e.g., $\left.f \in L^{\infty}\right), T_{0}^{0}(f)=T(f) *$. After developing the theory in $\S 2$, we will be able to show by example that this equality does not always hold. 
Proof of Lemma 1.2. $D_{0}^{0} \supset D_{0}$ is clear, and $D^{0} \supset D\left(T(f)^{*}\right)$ follows from the fact that $T^{0}(f)$ is a closed operator and contains $T_{0}(f)$. It remains to show $D_{0}^{0} \subset D\left(T(f)^{*}\right)$. This follows from $D\left(T(\bar{f})^{*}\right)=\left\{y^{+} \in H^{q}\right.$ : there exists a $z^{+}(\phi) \in H^{q}$ :

$$
\left(T(f) x^{+}, y^{+}\right)=\left(x^{+}, z^{+}\right)
$$

for all $\left.x^{+} \in D(T(\bar{f}))\right\}$ and from $\left(T(\bar{f}) x^{+}, y^{+}\right)=\left(x^{+}, T^{0}(f) y^{+}\right)$for all $x^{+} \in D(T(\bar{f}))$, $y^{+} \in D^{0}$. In order to verify this last equality, note that for $y^{+} \in D_{0}^{0}$,

$$
\left(x^{+}, T^{0}(f) y^{+}\right)=\left(x^{+}, f y^{+}\right)=\frac{1}{2 \pi} \int_{0}^{2 \pi} x^{+} \bar{f} \bar{y}^{+} d \phi .
$$

Let $x^{+} \bar{f}(\phi)=T(\bar{f}) x^{+}+\bar{x}_{1}^{+}(\phi) e^{-i \phi}$, so that

$$
\left(\bar{x}_{1}^{+} e^{-i \phi}\right) \bar{y}^{+}(\phi)=x^{+}\left(\bar{f} \bar{y}^{+}\right)-\left(T(\bar{f}) x^{+}\right) \bar{y}^{+} \in L^{1} .
$$

Now if the product of two $H^{1}$-functions belongs to $L^{1}$, then the product also belongs to $H^{1}$, so that $x_{1}^{+} y^{+} \in H^{1}$ and

$$
\int_{0}^{2 \pi} \bar{x}_{1}^{+} e^{-i \phi} \bar{y}^{+} d \phi=0, \quad \text { i.e., } \quad\left(x^{+}, T^{0}(f) y^{+}\right)=\left(T(\bar{f}) x^{+}, y^{+}\right)
$$

for all $x^{+} \in D(T(\bar{f})), y^{+} \in D_{0}^{0}$.

THEOREM 1.2. Let $2 \leqq q<\infty$ and let $f(\phi)$ be as in Theorem 1.1. Let $\alpha$, have the additional decomposition

$$
\alpha_{j}=\varepsilon_{j}+\delta\left(\theta_{j}\right), \quad \varepsilon_{j} \in Z, \quad-1 \leqq \delta\left(\theta_{j}\right)<0 .
$$

Let $d^{\prime}=\sum_{j=1}^{n} \varepsilon_{j}$, and for $0 \leqq \theta<2 \pi, \theta \neq \theta_{j}$, put $\delta(\theta)=-1$. Let

$$
\begin{gathered}
k^{\prime}=\sum_{0 \leqq \theta<2 \pi} M_{\gamma(\theta)}^{q}(f, \theta, \operatorname{conj} h(\phi)) \leqq \infty, \\
e=\sum_{0 \leqq \theta<2 \pi} \max \left[M_{-\delta(\theta)-1}^{q}(f, \theta,-\operatorname{conj} h), M_{-\delta(\theta)-1}^{q}(1 / f, \theta,-\operatorname{conj} h)\right] \leqq \infty .
\end{gathered}
$$

Then, if $\log |f(\phi)| \in L^{1}$ and $k^{\prime}, e<\infty$.

$$
\begin{aligned}
\max \left(d-k^{\prime}, 0\right) & =\operatorname{dim} N\left(T^{0}(\bar{f})\right) \geqq \operatorname{dim} N\left(T(f)^{*}\right) \geqq \operatorname{dim} N\left(T_{0}^{0}(\bar{f})\right) \\
& =\max \left(0, n-e-d^{\prime}\right) .
\end{aligned}
$$

Furthermore, $\log |f(\phi)| \notin L^{1}$ and $f \not \equiv$ imply that $R\left(T(f)^{*}\right) \neq \bar{R}\left(T(f)^{*}\right)=H^{q}$.

Proof. The proof of Theorem 1.1 may be applied without change to the case of $2 \leqq q, 2 \leqq p \leqq q$. This proves the statement involving $T^{0}(\bar{f})$. The conclusion for $T_{0}^{0}(\bar{f})$, in case $\log |f(\phi)| \notin L^{1}$ is proved precisely the same way as that for $T(f)$; cf. the first part of the proof of Theorem 1.1. We may, therefore, assume $\log |f(\phi)| \in L^{1}$. By Lemma 1.2, it suffices to prove $\operatorname{dim} N\left(T_{0}^{0}(\bar{f})\right)=\max \left(0, n-e-d^{\prime}\right)$. 
Consider, therefore, $f(\phi)=|f(\phi)| e^{-i(g(\phi)+h(\phi))}$. From the proof of Theorem 1.1,

$$
\operatorname{conj}[-g(\phi)]=\log \prod_{j=1}^{n}\left|1-\exp \left(i\left(\theta_{j}-\phi\right)\right)\right|^{-2 \alpha_{j}},
$$

$$
\begin{aligned}
\operatorname{conj} \arg \exp \left[-i \sum_{j=1}^{n}\left(\varepsilon_{j}-1\right)\left(\phi-\theta_{j}\right)\right] & =\operatorname{conj} \arg C e^{-i\left(d^{\prime}-n\right) \phi} \\
& =2 \log \prod_{j=1}^{n}\left|1-\exp \left(i\left(\phi-\theta_{j}\right)\right)\right|^{\varepsilon_{j}-1},
\end{aligned}
$$

$$
\begin{aligned}
\frac{1}{2} \operatorname{conj}\left[-g(\phi)+\arg C e^{-i\left(\alpha^{\prime}-n\right) \phi}\right] & =\log \prod_{j=1}^{n}\left|1-\exp \left(i\left(\phi-\theta_{j}\right)\right)\right|^{\varepsilon_{j}-\alpha_{j}-1} \\
& =\log \prod_{j=1}^{n}\left|1-\exp \left(i\left(\phi-\theta_{j}\right)\right)\right|^{-\delta\left(\theta_{j}\right)-1},
\end{aligned}
$$

so that, if

$$
F(\phi)=e^{G(\phi)}=C_{1} \prod_{j=1}^{n}\left|1-\exp \left(i\left(\phi-\theta_{j}\right)\right)\right|^{-(1+\delta(\theta))} \exp \frac{1}{2} i\left(g(\phi)+\left(d^{\prime}-n\right) \phi\right),
$$

then

$$
\begin{gathered}
F(\phi) \in H^{1}, \quad 1 / F(\phi) \in H^{\infty}, \\
\bar{F}(\phi) / F(\phi)=C_{2} e^{-i\left(d^{\prime}-n\right) \phi} e^{-i g(\phi)} .
\end{gathered}
$$

Following the procedure used in the proof of Theorem 1.1, we have

$$
\begin{aligned}
\operatorname{conj} \arg \exp \left[i \sum_{j=1}^{e}\left(\phi-\theta_{j}^{\prime}\right)\right] & =\operatorname{conj} \arg C e^{i e \phi} \\
& =-2 \log \prod_{j=1}^{e}\left|1-\exp \left(i\left(\phi-\theta_{j}^{\prime}\right)\right)\right| ; \\
& F_{1}(\phi)=\prod_{j=1}^{e}\left|1-\exp \left(i\left(\phi-\theta_{j}^{\prime}\right)\right)\right| e^{i e \phi / 2}
\end{aligned}
$$

satisfies $F_{1}(\phi) \in H^{\infty}$ and

$$
\bar{F}_{1}(\phi) / F_{1}(\phi)=e^{-t e \phi} \text {. }
$$

From (1.4), it follows that

$$
e^{-i h(\phi)} x_{2}^{+}(\phi)=\bar{x}_{2}^{+}(\phi)
$$

where $x_{2}^{+}(\phi)=1 / x_{1}^{+}(\phi), 1 / x_{2}^{+}(\phi) \in H^{b}$ for $1 \leqq b<\infty$. Combining (1.10), (1.11), (1.12), and (1.5), we obtain

$$
|f(\phi)| e^{-i(g(\phi)+h(\phi))} F(\phi) F_{1}(\phi) x_{2}^{+}(\phi) x^{+}(\phi) C_{2} e^{i\left(n-d^{\prime}-e\right) \phi}=\bar{F}(\phi) \bar{F}_{1}(\phi) \bar{x}_{2}^{+}(\phi) / \bar{x}^{+}(\phi) .
$$


It follows, by the same argument as that in the proof of Theorem 1.1, that

$$
\begin{gathered}
F_{3}(\phi)=F(\phi) F_{1}(\phi) x_{2}^{+}(\phi) x^{+}(\phi) \in H^{q}, \\
F_{3}(\phi) f(\phi) \in L^{q} .
\end{gathered}
$$

Hence $F_{3}(\phi) \in D_{0}^{0}$. This implies $\operatorname{dim} N\left(T_{0}^{0}(\bar{f})\right) \geqq n-e-d^{\prime}$.

If $x_{3}^{+}(\phi) \in D_{0}^{0}$ satisfies

$$
\bar{f}(\phi) x_{3}^{+}(\phi)=e^{-i \phi}\left(1+\bar{y}^{+}(\phi)\right),
$$

then, combining with (1.12) and (1.5),

$$
\frac{\bar{f}(\phi)}{|f(\phi)| e^{-i h(\phi)}} \frac{x_{3}^{+}(\phi)}{x_{2}^{+}(\phi) x^{+}(\phi)}=e^{-i \phi} \frac{1+\bar{y}^{+}(\phi)}{\bar{x}_{2}^{+}(\phi)} \bar{x}^{+}(\phi) .
$$

The function $X^{+}(\phi) \equiv x_{3}^{+}(\phi) / x_{2}^{+}(\phi) x^{+}(\phi)$ is of class $H^{1}$ since $x_{3}^{+} \in L^{q}$,

$$
1 /\left|x^{+}(\phi)\right|=|f(\phi)|^{1 / 2} \in L^{2 q}
$$

for $q \geqq 2$ and $1 / x_{2}^{+}(\phi) \in L^{b}$ for $1 \leqq b<\infty$. Also

$$
\left|X^{+}(\phi)\right|\left|x_{2}^{+}(\phi)\right| /|f(\phi)|^{1 / 2}=\left|x_{3}^{+}(\phi)\right| \in L^{q}
$$

and $f x_{3}^{+} \in L^{q}$, so that we get the following analogue of (1.8),

$$
\begin{gathered}
e^{-i g(\phi)} X^{+}(\phi)=e^{-i N \phi} \bar{Y}^{+}(\phi), \\
e^{-(1 / 2) \operatorname{conj} h(\phi)} X^{+}(\phi)|f(\phi)|^{ \pm 1 / 2} \in L^{q} .
\end{gathered}
$$

This together with the analogue (1.10) of (1.2) can be used to complete the proof of Theorem 1.2 by the arguments employed at the end of the proof of Theorem 1.1.

By noting $R(T)^{\perp}=N\left(T^{*}\right)$, we see that Theorem 1.2 gives a partial answer to the question: when is $T(f)$ one-to-one with a range of deficiency $n$ ?

Theorems 1.1 and 1.2 have the following corollary, which carries on the work of Hartman and Wintner [6] on Hermitian Toeplitz operators.

CoRollary 1.1. Let $f(\phi) \in L^{q}, \infty>q \geqq 2$, be real-valued. If $\log |f(\phi)| \in L^{1}$, assume that $f(\phi)$ is defined for all $\phi$ so that $\operatorname{sgn} f(\phi)(= \pm 1)$ is continuous except for a finite number of discontinuities, $\theta_{1}, \ldots, \theta_{2 n} \bmod 2 \pi$. Let

$$
k=\sum_{0 \leqq \phi<2 \pi} M_{-1 / 2}^{p}(f, \phi, 0) \text {. }
$$

Then (i) $\operatorname{dim} N(T(f))=\max (0, n-k)$,

(ii) $R(T(f))$ is dense in $H^{p}$,

(iii) $R(T(f)) \neq H^{p}$ if $n-k<0$.

In case $\log |f(\phi)| \notin L^{1}$ and $f \not \equiv 0$, then

(iv) $T(f)$ is one-to-one and $R(T(f)) \neq \bar{R}(T(f))=H^{p}$.

Actually the proof of (ii) uses only Lemma 1.2, so that it holds for any realvalued $f(\phi) \in L^{q}$. 
Proof of Corollary 1.1. Since $\arg f(\phi)=-(\pi / 2)(\operatorname{sgn} f(\phi)-1)$ it is clear that Theorem 1.1 applies with $h(\phi)=0$ and each $\gamma\left(\theta_{j}\right)=-1 / 2$, so that $n$ of the $\beta_{j}=1$ and $n$ of them are 0 . Thus $d=n$ and $\operatorname{dim} N(T)=\max (0, n-k)$ in (i). (iii) and (iv) also follow from Theorem 1.1.

(ii) follows from $T(f)^{*} \subset T^{0}(f)$ in Lemma 1.2, since, if $\bar{R}(T) \neq H^{p}$, then $N\left(T(f)^{*}\right) \neq\{0\}$, so $N\left(T^{0}(f)\right) \neq\{0\}$ and there is a $x^{+}(\phi) \in H^{q}$ satisfying

$$
f(\phi) x^{+}(\phi)=\bar{y}^{+}(\phi) e^{-i \phi} \text {. }
$$

But this means that $x^{+}(\phi) \in D(T) \subset H^{p}$. Hence, by applying $T$ to $e^{i N \phi} x^{+}$, for suitable values of $N$, we see that $1, e^{i \phi}, \ldots \in R(T)$, i.e., $\bar{R}(T)=H^{p}$, a contradiction.

REMARK. Duren [3] has conjectured that for $T \not \equiv 0$ a Toeplitz operator on the $l^{p}$ sequence spaces, the sets of $\lambda$ such that $\operatorname{dim} N(T-\lambda I)>0$ and $R(T-\lambda I)$ is not dense are disjoint. Actually, the proof of Duren's conjecture for $H^{p}$ spaces is quite simple and follows the lines of the proof [6] that a selfadjoint $T(f)$ has no point spectrum. In fact if $T(f) x^{+}(\phi)=0$, then applying $T$ to $e^{i N \phi} x^{+}$for $N$ sufficiently large, we see that $R(T)$ contains $1, e^{i \phi}, e^{2 i \phi}, \ldots$ and hence is dense in $H^{p}$.

Let

$$
k_{p}^{\prime \prime}=\sum_{0 \leqq \theta<2 \pi} M_{\gamma(\theta)}^{p}(f, \theta, 0) .
$$

In the next corollary, we follow the lines of [7], p. 74, (c), and show that, in certain cases, considerations of $\operatorname{dim} N(T)$ can be reduced to considerations of $d-k_{p}^{\prime \prime}$, i.e., of (1.1) with $H(\phi) \equiv 0$.

COROLLARY 1.2. Let $f(\phi) \in L^{q}$ satisfy the hypotheses of Theorem $1.1, \log |f(\phi)| \in L^{1}$ and $k, k^{\prime \prime}<\infty$.

(1) If $k_{p}^{\prime \prime}=k_{r}^{\prime \prime}$ for some $r>p$, then $\operatorname{dim} N(T(f)) \geqq d-k_{p}^{\prime \prime}$.

(2) If $k_{p}^{\prime \prime}=k_{r}^{\prime \prime}$ for some $r<p$, then $\operatorname{dim} N(T(f)) \leqq \max \left(0, d-k_{p}^{\prime \prime}\right)$.

(3) If there exists a $\psi(\phi)$ such that $h(\phi)$ has a bounded conjugate function, then $\operatorname{dim} N(T(f))=\max \left(0, d-k_{p}^{\prime \prime}\right)$.

Proof. For $p<r$, we have

$$
M_{\gamma(\theta)}^{r}(f, \theta, 0) \geqq M_{\gamma(\theta)}^{p}(f, \theta, 0),
$$

so that $k_{p}^{\prime \prime}=k_{r}^{\prime \prime}$ implies that each

$$
M_{\gamma(\theta)}^{r}(f, \theta, 0)=M_{\gamma(\theta)}^{p}(f, \theta, 0) \equiv M[\theta] .
$$

Hence

$$
(\phi-\theta)^{2 M[\theta]+2 \gamma} \mid f(\phi) \in L^{r / 2}(U),
$$

for all sufficiently small neighborhoods $U$ of $\theta$. Now, since $e^{\text {cons } h(\phi)} \in L^{b}$ for $1 \leqq b<\infty$, we have

$$
e^{\operatorname{conj} h(\phi)}(\phi-\theta)^{2 M[\theta]+2 \gamma} \mid f(\phi) \in L^{p / 2}(U) .
$$

Hence $M_{\gamma(\theta)}^{p}(f, \theta$, conj $h(\phi)) \leqq M_{\gamma(\theta)}^{p}(f, \theta, 0), k \leqq k_{p}^{\prime \prime}$, i.e., $d-k_{p}^{\prime \prime} \leqq d-k$. This proves (1). 
For (2), we have, from the proof of (1), that

$$
M_{\gamma(\theta)}^{r}(f, \theta, 0)=M_{\gamma(\theta)}^{p}(f, \theta, 0) .
$$

Thus,

$$
|\phi-\theta|^{2(M[\theta]-1-\gamma)} \mid f(\phi) \notin L^{r / 2}(U),
$$

for all neighborhoods $U$ of $\theta$. But now, if

$$
t(\phi)=e^{\operatorname{conj} h(\phi)}|\phi-\theta|^{2(M[\theta]-1-\gamma)} \mid f(\phi) \in L^{p / 2}(U),
$$

for a (sufficiently small) neighborhood $U$ of $\theta$, then

$$
e^{- \text {conj } h(\phi)} t(\phi)=|\phi-\theta|^{2(M[\theta]-1-\gamma)} \mid f(\theta) \in L^{r / 2}(U),
$$

for all $r<p$, contradicting (1.15). This proves $k \geqq k_{p}^{\prime \prime}$, hence (2).

For (3), $|\operatorname{conj} h(\phi)| \leqq C$ implies that $0<C_{1} \leqq e^{\operatorname{conj} h(\phi)} \leqq C_{2}$ so that (1.1) holds with $H(\phi)=$ conj $h(\phi)$ if and only if (1.1) holds with $H(\phi) \equiv 0$. Thus $k=k_{p}^{\prime \prime}$, proving (3).

Corresponding estimates may be obtained from the conclusion of Theorem 1.2.

2. Nonselfadjoint $T(f)$. Applying Theorem 1.1 to $f(\phi)=G(\phi) \pm i$, we obtain the following conditions for $T(G): H^{2} \rightarrow H^{2}$ to be selfadjoint, when $G(\phi)$ is real-valued.

THEOREM 2.1. Let $G(\phi) \in L^{2}$ be real-valued. Suppose that $G(\phi)$ is continuous, except for jump discontinuities. Suppose, also, that (after adding a measurable, bounded, real-valued function to $G$, if necessary) the functions

$$
h_{j}(\phi)=\psi_{j}(\phi)-g_{j}(\phi), \quad j=1,2
$$

can be extended as continuous, periodic functions of period $2 \pi$, where

$$
g_{j}(\phi)=\sum_{s=0}^{n} \alpha_{s j} J\left(\phi-\phi_{s}\right), \quad\left|\alpha_{s j}\right| \leqq 1 / 2, \quad j=1,2,
$$

and

$$
0<-\psi_{2}(\phi)=\psi_{1}(\phi)=\arg (G(\phi)+i)<\pi .
$$

From among the $\left\{\phi_{s}\right\}$, let $\phi_{1}^{+}, \ldots, \phi_{\mu}^{+}, \phi_{1}^{-}, \ldots, \phi_{v}^{-}, \theta_{1}^{+}, \ldots, \theta_{s}^{+}, \theta_{1}^{-}, \ldots, \theta_{t}^{-}$be the points at which $g(\phi)$ has a discontinuity of magnitude $\pi$. Let

$$
\begin{array}{ll}
-G\left(\xi_{j}^{+}-0\right)=G\left(\xi_{j}^{+}+0\right)=\infty & \text { for } \xi=\theta, \phi ; \\
-G\left(\xi_{j}^{-}+0\right)=G\left(\xi_{j}^{-}-0\right)=\infty & \text { for } \xi=\theta, \phi ;
\end{array}
$$

$$
\exp \left(\operatorname{conj} h_{2}(\phi)\right) /\left[(1+|G(\phi)|)\left(\phi-\phi_{j}^{+}\right)\right] \in L^{1}(U), \quad j=1, \ldots, \mu,
$$

for some neighborhood $U$ of $\phi_{j}^{+}$;

$$
\exp \left(\operatorname{conj} h_{1}(\phi)\right) /\left[(1+|G(\phi)|)\left(\phi-\phi_{j}^{-}\right)\right] \in L^{1}(U), \quad j=1, \ldots, \nu,
$$

for some neighborhood $U$ of $\phi_{j}^{-}$;

(e) (c) and (d) do not hold with $\phi_{j}^{+}, \phi_{j}^{-}$replaced by $\theta_{j}^{+}, \theta_{j}^{-}$, respectively. 
Then, the deficiency indices of $T(G)^{*}$ are $\operatorname{dim} N(T(G)+i I)=\max (0, \nu-s)$; $\operatorname{dim} N(T(G)-i I)=\max (0, \mu-t)$.

Proof. By a theorem of Kreĭn (cf. [10, p. 150]), we may add a bounded, selfadjoint operator to $T(G)$ without changing the deficiency indices of $T(G)^{*}$. If $G_{0}$ is a bounded, real-valued function, we may, therefore, replace $G$ by $G+G_{0}$ and $T(G)$ by $T\left(G+G_{0}\right)=T(G)+T\left(G_{0}\right)$ and assume $h_{j}=\arg (G \pm i)-g_{j}$ is continuous. Now if $\alpha_{11}, \alpha_{21}, \ldots, \alpha_{N 1} ; \alpha_{12}, \alpha_{22}, \ldots, \alpha_{M 2}$ are the $\alpha_{s j}$ which satisfy $\left|\alpha_{s j}\right|<\frac{1}{2}$, $\alpha_{s j} \geqq 0$, then it is clear that for $G+i$, we have $d=\nu+t+N, k=s+t+N, d-k=\nu-s$. Similarly, for $G-i, d=\mu+s+M, k=s+t+M, d-k=\mu-t$.

In [7] it was shown that, under certain conditions, the deficiency indices of $T(G)^{*}$ depend only upon the number of jumps of $G(\phi)$ from $-\infty$ to $\infty$ and from $\infty$ to $-\infty$. The next corollary shows that in our (more general) situation, these numbers still determine whether $T(G)$ has selfadjoint extensions.

Corollary 2.1. Let $G(\phi) \in L^{2}$ satisfy the conditions of Theorem 2.1. Let $T(G)$ be nonselfadjoint and let

$$
\begin{aligned}
& n_{+}=\text {number of jumps of } G(\phi) \text { from }-\infty \text { to } \infty, \\
& n_{-}=\text {number of jumps of } G(\phi) \text { from } \infty \text { to }-\infty .
\end{aligned}
$$

Then $T(G)^{*}$ has selfadjoint extensions if and only if $n_{+}=n_{-}$.

Proof. In the notation of Theorem $2.1, n_{+}=\mu+s, n_{-}=\nu+t$, and $n_{+}=n_{-}$if and only if $\mu+s=\nu+t$, i.e., $\mu-t=\nu-s$.

The next corollary generalizes (i)-(iv) of [7, p. 74, (c)]. In [8], Ismagilov proved that $T(G)$ is selfadjoint if every point of $[0,2 \pi]$ has a neighborhood in which $G$ is half-bounded. Actually, Ismagilov's theorem can be obtained from the results of [7], by the method of (a), (p. 74 of [7]). We will show below that the converse to Ismagilov's theorem is false. At the same time, we answer affirmatively the question raised by Hartman in [7], whether or not $T(G)$ can be selfadjoint if $\mu+s>0$ or $\nu+t>0$; one need only take $\sigma^{\prime}=s \geqq \nu, \tau^{\prime}=t \geqq \mu$ in (3) below.

COROLlaRY 2.2. Let $G(\phi) \in L^{2}$ be real-valued and satisfy the conditions of Theorem 2.1. Let $\xi_{1}^{+}, \ldots, \xi_{\sigma}^{+} ; \xi_{1}^{-}, \ldots, \xi_{\tau}^{-}$be the points such that $G(\phi)$ jumps from $-\infty$ to $\infty$ and from $\infty$ to $-\infty$ respectively.

(1) If $\sigma=0$, then $\operatorname{dim} N(T(G)-i I)=0$; if $\tau=0$, then $\operatorname{dim} N(T(G)+i I)=0$.

(2) If

$$
1 /[(1+|G(\phi)|)(\phi-\xi)] \in L^{p}
$$

for some $p>1$, for $\xi_{j}=\xi_{j}^{+}, j=1, \ldots, \mu^{\prime}$ and for $\xi_{j}=\xi_{j}^{-}, j=1, \ldots, \nu^{\prime}$, then

$$
\operatorname{dim} N(T(G)+i I) \geqq \nu^{\prime}-\left(\sigma-\mu^{\prime}\right)
$$

and

$$
\operatorname{dim} N(T(G)-i I) \geqq \mu^{\prime}-\left(\tau-\nu^{\prime}\right)
$$


(3) If (2.1) fails to hold, for some $p<1$, for $\xi_{j}=\xi_{j}^{+}, j=1, \ldots, \sigma^{\prime}$ and for $\xi_{j}=\xi_{j}^{-}$, $j=1, \ldots, \tau^{\prime}$, then

$$
\operatorname{dim} N(T(G)+i I) \leqq \max \left(\left(\tau-\tau^{\prime}\right)-\sigma^{\prime}, 0\right),
$$

and

$$
\operatorname{dim} N(T(G)-i I) \leqq \max \left(\left(\sigma-\sigma^{\prime}\right)-\tau^{\prime}, 0\right) .
$$

(4) If there exist functions $\psi_{j}, j=1,2$, such that the functions $h_{j}(\phi), j=1,2$, have bounded conjugate functions; if (2.1) with $p=1$ holds for $\mu$ choices of the $\xi_{j}^{+}$and $\nu$ choices of the $\xi_{j}^{-}$, and if (2.1) with $p=1$, fails to hold for the remaining $s=\sigma-\mu$ choices of $\xi_{j}^{+}$and $t=\tau-\nu$ choices of $\xi_{j}^{-}$, then $\operatorname{dim} N(T(G)+i I)=\max (0, \nu-s)$; $\operatorname{dim} N(T(G)-i I)=\max (0, \mu-t)$.

Proof. (1) is clear from Theorem 2.1. In order to obtain (2), we note that (c) and (d) must be satisfied for points satisfying (2.1) with $p>1$. Thus, $\mu \geqq \mu^{\prime}, \nu \geqq \nu^{\prime}$ and hence $s=\sigma-\mu \leqq \sigma-\mu^{\prime}, t=\tau-\nu \leqq \tau-\nu^{\prime}$, so that $\mu-t \geqq \mu^{\prime}-\left(\tau-\nu^{\prime}\right), \nu-s \geqq \nu^{\prime}-\left(\sigma-\mu^{\prime}\right)$. This proves (2).

For (3), we note that if $\xi_{j}$ fails to satisfy (2.1) for some $p<1$, then it cannot satisfy (c) or (d) of Theorem 2.1. Thus $s \geqq \sigma^{\prime}, t \geqq \tau^{\prime}$, (3) follows. (4) is clear.

Hartman and Wintner proved, in [6], that, if $T(G)$ is selfadjoint, then $T(G)$ has no point spectrum. Combining this result with our Corollary 1.1, we obtain the following somewhat different condition for $T(G)$ to be nonselfadjoint:

THeOREM 2.2. Suppose $G(\phi) \in L^{2}$ is real-valued. Let there exist a finite number of points $0 \leqq \theta_{1}<\cdots<\theta_{2 n} \leqq 2 \pi$ such that on $\left(\theta_{j}, \theta_{j+1}\right), j=1, \ldots, n-1$ (and on $\left(0, \theta_{1}\right) \cup\left(\theta_{2 n}, 2 \pi\right)$ in case $0<\theta_{1}$ or $\left.\theta_{2 n}<2 \pi\right) G(\phi)$ is either bounded from above or bounded from below. Let $k$ be the number of points $\theta_{\text {, such that }}$

$$
1 /\left[(1+|G(\phi)|)\left(\theta_{j}-\phi\right)\right] \notin L^{1} .
$$

Let $n>k$ then $T(G)$ is not selfadjoint and

$$
\min [\operatorname{dim} N(T(G)+i I), \operatorname{dim} N(T(G)-i I)] \geqq n-k .
$$

Proof. We may assume that either $G(\phi) \geqq \varepsilon>0$ or $G(\phi) \leqq-\varepsilon<0$ on each interval in question, for otherwise, by the theorem of Krein [10] mentioned above, a bounded function may be added to $f(\phi)$ without changing the deficiency indices of $T(G)^{*}$ or the conditions of the theorem. Corollary 1.1 then implies that $T(G)$ has a nullspace of dimension at least $n-k$, so that $T(G)$ is not selfadjoint.

In order to verify (2.2), we first note that $0 \notin \mathrm{pt}$. sp. $T^{*}=T(G)^{*}$ (Corollary 1.1). Now it is clear that $(\alpha)$ of [5] generalizes to the case of unequal deficiency indices. Thus, if $x \in N(T)$ has the decomposition $x=x_{0}+v_{1}+v_{2}$, where $x_{0} \in D\left(T^{*}\right)$, $v_{1} \in N(T-i I), v_{2} \in N(T+i I)$, then $\left\|v_{1}\right\|=\left\|v_{2}\right\|$. Now if

$$
\operatorname{dim} N(T+i I)=m<\operatorname{dim} N(T-i I)
$$


and if there are $m+1$ solutions $x_{j}=x_{j 0}+v_{j 1}+v_{j 2}$ of $T x_{j}=0$, let $\sum_{j=1}^{m+1} \alpha_{j} v_{j 2}=0$. Then $x=\sum_{j=1}^{m+1} \alpha_{j} x_{j}=\sum_{j=1}^{m+1} \alpha_{j} x_{j 0}+\sum_{j=1}^{m+1} \alpha_{j} v_{j 1}$ satisfies $T x=0$ and hence

$$
\left\|\sum_{j=1}^{m+1} \alpha_{j} v_{j 1}\right\|=\left\|\sum_{j=1}^{m+1} \alpha_{j} v_{j 2}\right\|=0 .
$$

Thus $x=\sum_{j=1}^{m+1} \alpha_{j} x_{j 0} \in D\left(T^{*}\right)$. But since $\operatorname{dim} N\left(T^{*}\right)=0, x=0$. This proves (2.2) and hence Theorem 2.2.

REMARK. On the basis of Theorem 2.1 , we may conclude that equality does not always hold in $T_{0}^{0}(f) \subset T(f)^{*}$ in Lemma 1.2. In fact, if $G(\phi) \in L^{2}$ is real-valued, satisfies Theorem 2.1 and $\mu-s, \nu-t \leqq 0$, but $\mu, s, \nu, t<\infty$, then the function $K(\phi)=x^{+} x_{1}^{+} F F_{1}$ of (1.6) surely satisfies $K(\phi) \in D(T(G)), K(\phi) \notin D_{0}^{0}$, but by Theorem 2.1, $T(G)$ is selfadjoint, so $K(\phi) \in D\left(T(G)^{*}\right)-D_{0}^{0}$.

\section{REFERENCES}

1. A. Calderón, F. Spitzer and H. Widom, Inversion of Toeplitz matrices, Illinois J. Math. 3 (1959), 490-498.

2. A. Devinatz, Toeplitz operators on $H^{2}$-spaces, Trans. Amer. Math. Soc. 112 (1964), 304-317.

3. P. L. Duren, On the spectrum of a Toeplitz operator, Pacific J. Math. 14 (1964), 21-29.

4. P. Hartman and A. Wintner, On the spectra of Toeplitz's matrices, Amer. J. Math. 72 (1950), 359-366.

5. P. Hartman, On the essential spectra of symmetric operators, Amer. J. Math. 75 (1953), 229-240.

6. P. Hartman and A. Wintner, The spectra of Toeplitz's matrices, Amer. J. Math. 76 (1954), 867-882.

7. P. Hartman, Unbounded Toeplitz matrices, Amer. J. Math. 85 (1963), 59-78.

8. R. S. Ismagilov, The spectrum of Toeplitz matrices, Dokl. Akad. Nauk SSSR 149 (1963), 769-774 = Soviet Math. Dokl. 4 (1963), 462-465.

9. M. G. Kreìn, Integral equations on a half-line with kernel depending on the difference of the arguments, Uspehi Mat. Nauk 13 (1958), no. 5 (83), 3-120; English transl., Amer. Math. Soc. Transl. (2) 22 (1962), 163-288.

10. M. A. Neumark, Lineare Differentialoperatoren, Akademie-Verlag, Berlin, 1960.

11. G. Szegö, Ueber die Randwerte einer analytischen Funktion, Math. Ann. 84 (1931), 232-244.

12. H. Widom, Inversion of Toeplitz matrices. II, Illinois J. Math. 4 (1960), 88-99.

THE JOHNS HOPKINS UNIVERSITY, BALTIMORE, MARYLAND 